\title{
Electroluminescence Investigation of the Lateral Field Distribution in AlGaN/GaN HEMTs for Power Applications
}

\author{
M. Baeumler*, V.M. Polyakov, F. Gütle, M. Dammann, F. Benkhelifa, P. Waltereit, \\ R. Reiner, S. Müller, M. Wespel, R. Quay, M. Mikulla, J. Wagner and O. Ambacher \\ Fraunhofer-Institut für Angewandte Festkörperphysik, Tullastr. 72, 79108 Freiburg, Germany
}

\begin{abstract}
The lifetime and stability of $\mathrm{AlGaN} / \mathrm{GaN}$ heterostructure field effect transistors at high power levels can be enhanced by introducing field plates to reduce electric field peaks in the gate-drain region. Simulations of the electric field distribution along the channel using the 2D ATLAS software from Silvaco indicate that above a characteristic drain source voltage three spatially separated electric field peaks appear, one located at the drain-side edge of the gate foot, one at the end of the drain-sided gate field plate, and one at the end of the source shield field plate. The close correlation between lateral electric field and the electroluminescence due to hot electron related intra-band transitions can be very helpful when optimizing the electric field distribution in high power devices. Electroluminescence microscopy images of devices with gate and source shield field plate reveal the peaks located at the locations of enhanced electric field. By studying the voltage dependence of the electroluminescence peaks the influence of the field plates on the electric field distribution in source drain direction can be visualized.
\end{abstract}

DOI: $10.12693 /$ APhysPolA.125.982

PACS: 78.60.Fi, 85.30.De, 85.30.Tv, 85.40.Bh, 85.40.Qx, 73.40.-c, 73.90.+f, 73.61.Ey, 73.50.Mx

\section{Introduction}

The use of $\mathrm{AlGaN} / \mathrm{GaN}$ high electron mobility transistors (HEMTs) for power applications requires not only excellent performance but also long lifetimes and high voltage capability. Consequently, in-depth reliability assessments in conjunction with failure analysis using electroluminescence microscopy (ELM) have to be performed [1]. The application of ELM is not only limited to the localization of degraded areas but also allows to assess local variations of electrical parameters as leakage current [2-4], threshold voltage [5], and peak electric field $[6,7]$.

Simulations of the electric field distribution in the gate drain region of $\mathrm{AlGaN} / \mathrm{GaN}-\mathrm{HEMTs}$ indicate that the electric field is peaked at the drain-sided edge of the foot of the gate electrode $[6,8-10]$. This is consistent with transmission electron microscopy (TEM) investigations $[11,12]$ where most of the damage has been observed to be located at this position. The lifetime and stability of AlGaN/GaN HEMTs at high power levels can be enhanced by introducing a gate field plate to reduce the peak electric field strength at the drain-side gate foot edge [13]. Simulations of the electric field distribution along the channel $F_{x}$ using the 2D ATLAS software from Silvaco [14] indicate that above a characteristic drainsource voltage two spatially separated peaks appear, one located at the drain-side edge of the gate foot, and one at the end of the drain-sided gate field plate $[7,8]$.

As the electroluminescence (EL) in AlGaN/GaN HEMTs is related to hot electrons accelerated in the elec-

\footnotetext{
* corresponding author; e-mail:

martina.baeumler@iaf.fraunhofer.de
}

tric field in the source-drain direction $F_{x} \|$, the technique offers a unique possibility to visualize the spatial distribution of $F_{x}$. By imaging the EL of devices with sufficiently large gate field plate length through the substrate, two spatially separated EL intensity maxima have been resolved, one located at the drain-side edge of the gate foot and the other at the end of the gate field plate [7]. Their positions correlate well with the electric field maxima extracted from the simulations. By varying the drainsource voltage and plotting the integrated EL intensity as a function of the electric field in the source-drain direction $F_{x}$, a threshold electric field for EL emission in the range of $100-150 \mathrm{kV} \mathrm{cm}^{-1}$ has been extracted. We proposed that radiative inter-valley transitions within the conduction band are the dominant EL-generating mechanism. Above the threshold electrons gain sufficient energy to be injected into the higher-lying conduction band satellite valleys, from where they relax back into the $\Gamma$ valley emitting a photon [7].

Additional homogenization of the electric field can be achieved by introducing source shield field plates [13]. Figure 1 presents results from a long term high temperature reverse bias (HTRB) step stress test on eight $\Gamma$-shaped gate AlGaN/GaN-HEMTs on $\mathrm{SiC}$ substrate [15]. The devices were die-attached to TO220 metal packages and stressed at $150^{\circ} \mathrm{C}$ in off-state. Devices with and without source shield field plate have been compared. During the stress test the drain voltage was increased from $200 \mathrm{~V}$ every $100 \mathrm{~h}$ by $100 \mathrm{~V}$ up to a maximum stress voltage of $600 \mathrm{~V}$. After each increase the

"For a detailed discussion of the different models for the EL emission and the corresponding references the reader is referred to Ref. [7]. 
relative change of the dynamic $R_{\text {on }}$ resistance has been determined at room temperature. The dynamic $R_{\text {on }}$ was defined as the relative change of the on-state resistance before and after an off-state pulse at a drain voltage of $100 \mathrm{~V}$ for $15 \mathrm{~s}$. The on-state resistance was measured at a gate voltage of $1 \mathrm{~V}$ and a drain current of $0.1 \mathrm{~A}$. The waiting time after the off-state pulse was $0.36 \mathrm{~s}$. Figure 1 shows that the shield reduces the degradation of the dynamic $R_{\text {on }}$ resistance during stress. The higher stability of the dynamic $R_{\text {on }}$ increase in devices with shield is considered to be due to a reduction of the electric field peak at the gate terminated field plate in the gate drain region. As a consequence the formation of slow traps in the AlGaN barrier responsible for the increase the dynamic $R_{\text {on }}$ resistance is likely to be reduced [16].

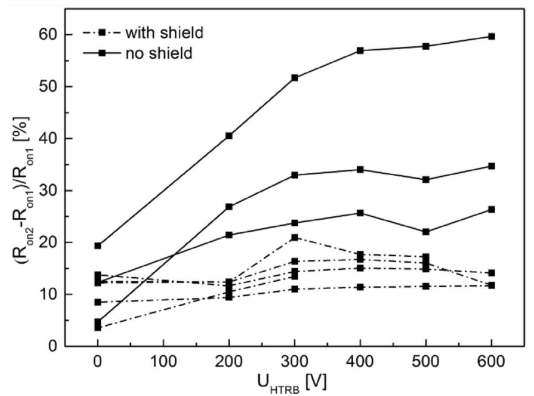

Fig. 1. Increase of dynamic $R_{\text {on }}$ during HTRB step stress test at a base plate temperature of $150{ }^{\circ} \mathrm{C}$ of packaged GaN HEMTs on $\mathrm{SiC}$ substrate with an increase $\Delta U_{\text {HTRB }}=100 \mathrm{~V}$ every $100 \mathrm{~h}$. After each step the dynamic $R_{\text {on }}$ was measured $0.36 \mathrm{~s}$ after an off-state pulse at $100 \mathrm{~V}$ for $15 \mathrm{~s}$ [15].

The close correlation between lateral electric field and EL can be very useful when optimizing the electric field distribution in high power devices. In this contribution we will present results from ELM and simulations of the electric field distribution in source drain direction of devices with shield and compare those to results on devices without shield. We will show that ELM offers a unique possibility to visualize the influence of the shield on the electric field distribution $F_{x}$.

\section{Experimental details}

AlGaN/GaN HEMTs with a drain-sided gate field plate (GFP) and a source shield field plate (shield) for high voltage applications have been investigated. Their relative lengths are $L_{\mathrm{GFP}} / L_{\mathrm{G}}=2$ and $L_{\mathrm{S}} / L_{\mathrm{G}}=4$ respectively, with $L_{\mathrm{G}}=2 \mu \mathrm{m}$ being the gate length. All HEMTs possess two gate fingers (GFs) with a gate width of $200 \mu \mathrm{m}$ each. The presented transistors are from one typical HEMT structure, which was grown on the semi-insulating $4 \mathrm{H}-\mathrm{SiC}$ substrate. The growth started with an AlN nucleation and a $1.8 \mu \mathrm{m}$ GaN buffer layer. As a barrier, a $27 \mathrm{~nm}$ thick $\mathrm{Al}_{0.23} \mathrm{Ga}_{0.77} \mathrm{~N}$ layer was grown, followed by a $3 \mathrm{~nm}$ GaN cap. Standard ohmic and the Schottky contacts were used for the drain/source and gate, respectively. Further information on the device fabrication can be found in [17].
On-wafer backside viewed EL images were recorded through the substrate by a Peltier-cooled Si-CCD camera (Andor DU934 N-BRD). This technique allows to spatially resolve the EL maxima at the gate foot which, in the case of top-view inspection, are shaded by the contact and the field plate metallization. The experiments were performed for on-state conditions at a gate voltage of $U_{\mathrm{G}}=-2.7 \mathrm{~V}$, about $1 \mathrm{~V}$ above threshold. $U_{\mathrm{DS}}$ was swept from 1 to $99 \mathrm{~V}$ while the EL image, the drain current $\left(I_{\mathrm{D}}\right)$ and the gate current $\left(I_{\mathrm{G}}\right)$ were recorded. Further details on the experiment can be found in Ref. [7].

\section{Results}

Figure 2 shows the backside viewed EL images of an $\mathrm{AlGaN} / \mathrm{GaN}$ HEMT with two gate fingers, recorded in on-state $\left(U_{\mathrm{G}}=-2.7 \mathrm{~V}\right)$ at different $U_{\mathrm{DS}}=20,45$, and $99 \mathrm{~V}$. The EL images are overlaid to the visible image recorded in transmission mode, with the metal contacts appearing in black. At $U_{\mathrm{DS}}=20 \mathrm{~V}$ both GFs reveal one EL intensity (ELI) maximum located along the drainside edge of the gate foot (ELIF). In the ELM image recorded at $45 \mathrm{~V}$ the appears at the end of the gate field plate a second ELI maximum (ELIP). At $U_{\mathrm{DS}}=99 \mathrm{~V}$ a third ELI maximum (ELIS) can be observed located at the end of the shield, which is missing for devices without shield [7].
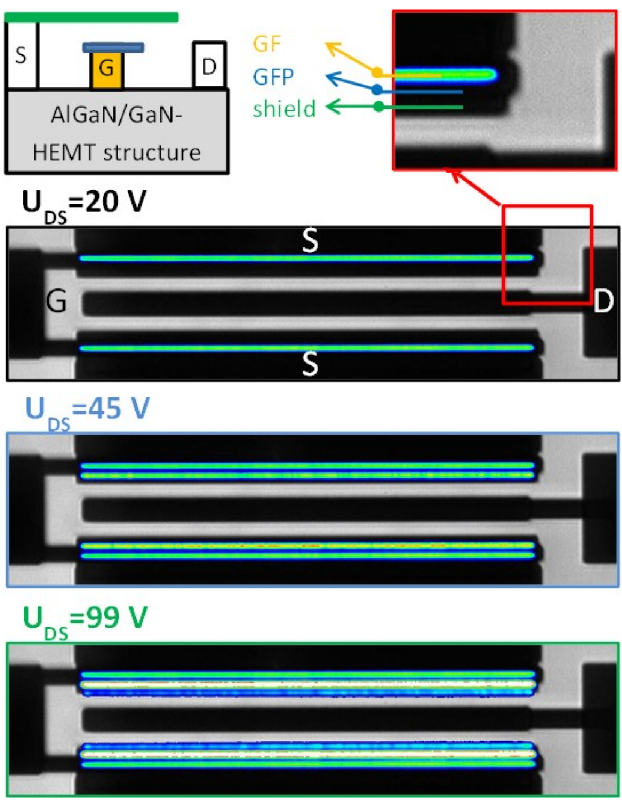

Fig. 2. EL images (color) of an AlGaN/GaN HEMT recorded at $U_{\mathrm{G}}=-2.7 \mathrm{~V}$ and different $U_{\mathrm{DS}}$ overlaid to the transmission image (black and white). The metallic contacts gate $(\mathrm{G})$, drain (D) and source $(\mathrm{S})$ appear black. The location of the GF, GFP, and source shield field plate (shield) are indicated in the blow-up.

Figure 3a shows ELI profiles recorded for $10 \mathrm{~V}<U_{\mathrm{DS}}<$ $99 \mathrm{~V}$, plotted versus the lateral position in the sourcedrain-direction given in units of $L_{\mathrm{G}}$. The profiles were generated by integrating ELI over the gate width. The 
population of the conduction band satellite valleys and hence the EL intensity is determined by the magnitude of the electric field and the resulting electron density along the channel [18]. The simulated electric field distribution in the source-drain-direction is shown in Fig. 3b. The positions of the three EL peaks correlate well with those of the electric field peaks at GF, GFP and the shield (see Fig. 2). Let us note that the EL peaks are broadened by the limited spatial resolution of the EL microscope.

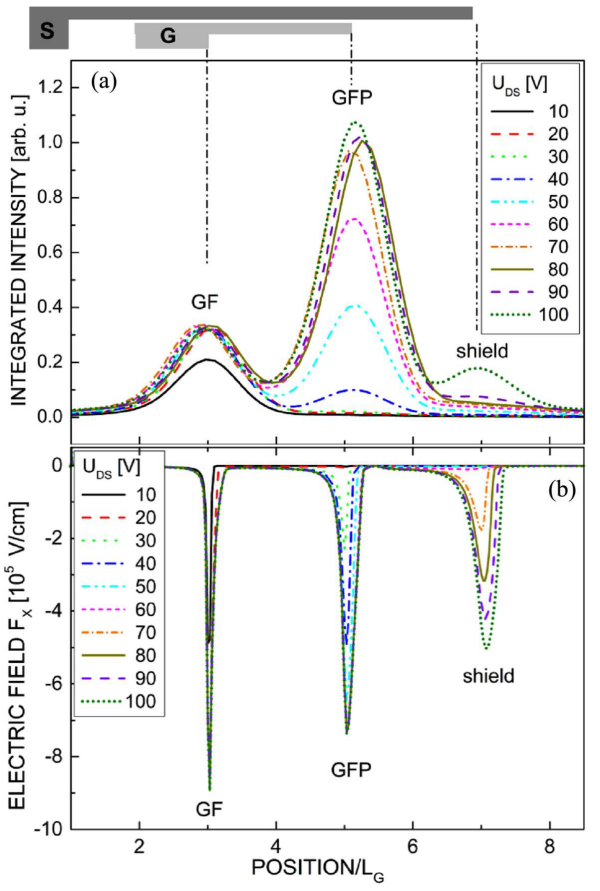

Fig. 3. (a) ELI profiles generated by integrating the ELI over the gate width and (b) electric field distribution along the source drain direction for $10 \mathrm{~V}<U_{\mathrm{DS}}<$ $99 \mathrm{~V}$, plotted versus the lateral position given in units of $L_{\mathrm{G}}$.

The influence of the two field plates GFP and the shield on the electric field distribution $F_{x}$ can be summarized as follows. Up to $U_{\mathrm{DS}}=20 \mathrm{~V}$ only the $F_{x}$ maxima at the GF can be detected. Above $20 \mathrm{~V}$ a second $F_{x}$ is located at the GFP edge while $F_{x}$ at the GF edge saturates. Above $60 \mathrm{~V}$ a third $F_{x}$ maxima at the shield edge appears and $F_{x}$ at the GFP edge saturates. The corresponding EL maxima at the GFP and shield edge can be detected above $30 \mathrm{~V}$ and $80 \mathrm{~V}$.

The integrated area IELI under the peaks is plotted in Fig. 4a as a function of $U_{\mathrm{DS}}$ (closed symbols). The open symbols represent the results for a transistor from the same wafer but without shield. The ELI was normalized to $I_{\mathrm{D}}$ taking account for a slight decrease of $I_{\mathrm{D}}$ due to self-heating at higher $U_{\mathrm{DS}}$.

The electric field maxima $F_{x}$ extracted from the simulations for the corresponding $U_{\mathrm{DS}}$ are plotted in Fig. $4 \mathrm{~b}$ using the same symbols. The intensity of the EL peak at the drain-side edge of the gate foot (IELIF) saturates above $10 \mathrm{~V}$ due to the saturation of $F_{x}$ (Fig. $3 \mathrm{~b}$ and $4 \mathrm{~b}$ ).

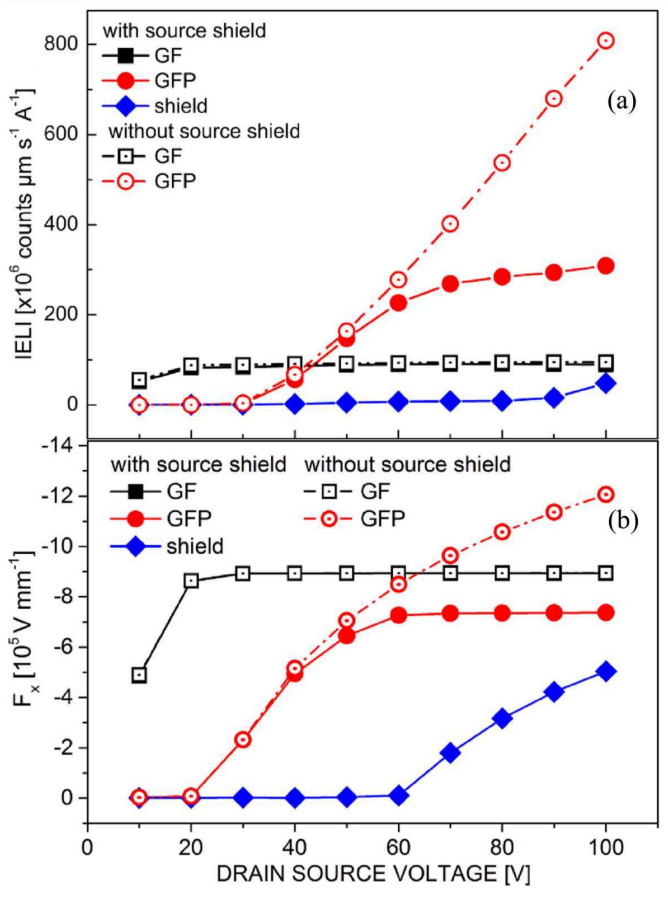

Fig. 4. (a) IELI at the drain-side gate foot edge (squares), the edge of the gate field plate (circle) and the edge of the source shield field plate (diamond) for a device with (closed symbols) and without (open symbols) shield plotted as a function of $U_{\mathrm{DS}}$. (b) Electric field maxima $F_{x}$ as extracted from the simulations in Fig. $3 \mathrm{~b}$ for the corresponding $U_{\mathrm{DS}}$.

The EL at the end of the gate field plate (IELIP) sets in for $U_{\mathrm{DS}}>30 \mathrm{~V}$, increasing almost linearly with $V_{\mathrm{DS}}$, with IELIP exceeding IELIF for $U_{\mathrm{DS}}=42 \mathrm{~V}$. For devices with shield IELIP starts to saturate above $U_{\mathrm{DS}}=60 \mathrm{~V}$, just then when the $F_{x}$ peak at the shield edges starts to appear resulting in a saturation of the $F_{x}$ peak at the GFP edge (Fig. $3 \mathrm{~b}$ and $4 \mathrm{~b}$ ). The IELI at the shield (IELIS) starts to increase above $80 \mathrm{~V}$. The delayed appearance of the EL peaks is caused by the threshold behavior of the EL signal described in detail in Ref. [7]. The threshold field for EL emission was found to be in the range of $100-150 \mathrm{kV} \mathrm{cm}^{-1}$.

The results in Fig. 4 present an experimental proof for the homogenization of the electric field distribution in the gate drain region by introducing field plates into the design of the device. EL microscopy allows us to monitor the changes in the field distribution by evaluating the integrated EL intensity as a function of $U_{\mathrm{DS}}$. However, care has to be taken when comparing the absolute EL intensity values with the electric field strength. When comparing the two locations at the GF and the GFP, the IELI at the GFP is enhanced with respect to the corresponding $F_{x}$ values. As mentioned above, the EL is attributed to hot electrons in the channel, accelerated in the electric field. Following our suggestion from Ref. [7] that the EL emission from AlGaN/GaN HEMTs is dominated by radiative inter-valley electron transitions, the 
IELI is directly related to the magnitude and width of the electric field peak (Fig. 3b). The increased width of the $F_{x}$ peak at the position of the GFP (as shown in Fig. $3 b$ ) promotes a larger fraction of channel electrons to occupy the satellite valleys when travelling through the high electric field region located at the field plate edge. As a result, we observe an enhancement of the EL emission with increasing $U_{\mathrm{DS}}$ at this location in the channel.

It should be noted, as follows from simulations, that the lateral electric field distribution dramatically depends on the trap density at the $\mathrm{GaN} / \mathrm{SiN}$ passivation layer interface. The latter is closely related to the polarization induced charge $\sigma_{\text {pol }}$ at the AlGaN/GaN interface [19]. In our simulations we used donor-like traps with the concentration equal to $\sigma_{\text {pol }}$. The assumption is validated by the results presented in this paper. However, a different passivation method might result in a variation of the trap concentration [20] which can be estimated by a comparative study with our technique.

\section{Conclusion}

We have investigated the influence of different field plate configurations on the electric field distribution in AlGaN/GaN HEMTs by monitoring the EL emission. For HEMT structures with a long gate and source shield field plate, we have observed three peaks in the ELI distribution along the channel underneath the drain side of the gate finger and the source shield field plate. The location of these three ELI peaks perfectly correlate with the simulated lateral profile of the electric field which reveals three high electric field regions, namely at the drain-sided gate foot and the field plate edges, respectively. By investigating the EL emission in dependence on the drain-source voltage, we were able to monitor the homogenization of the electric field distribution in the gate drain region by using field plates.

\section{Acknowledgments}

The authors are very grateful to the staff of the Department of Device Processing at Fraunhofer-Institut für Angewandte Festkörperphysik (IAF) for expert device fabrication.

\section{References}

[1] M. Dammann, H. Czap, J. Rüster, M. Baeumler, F. Gütle, P. Waltereit, F. Benkhelifa, R. Reiner, M. Cäsar, H. Konstanzer, S. Müller, R. Quay, M. Mikulla, O. Ambacher, in: IEEE Int. Integrated Reliability Workshop, IIRW 2012, Final Report, 2012, Stanford Sierra Conference Center, IEEE, Fallen Leaf Lake, CA, New York, 2012, p. 105.

[2] M. Baeumler, F. Gütle, V. Polyakov, M. Cäsar, M. Dammann, H. Konstanzer, W. Pletschen, W. Bronner, R. Quay, P. Waltereit, M. Mikulla. O. Ambacher, F. Bourgeois, R. Bethash, K. Riepe, P.J. van der Wel, J. Klappe, T. Rödle, J. Electron. Mater. 39, 756 (2010).

[3] D.A. Cullen, D.J. Smith, A. Passaseo, V. Tasco, A. Stocco, M. Meneghini, G. Meneghesso, E. Zanoni, IEEE Trans. Dev. Mater. Reliab. 13, 126 (2013).

[4] A. Zanandrea, E. Bahat-Treidel, F. Rampazzo, A. Stocco, M. Meneghini, E. Zanoni, O. Hilt, P. Ivo, J. Wuerf, G. Meneghesso, Microelectron. Reliab. 52, 2426 (2012)
[5] F. Gütle, M. Baeumler, M. Dammann, M. Cäsar, H. Walcher, P. Waltereit, W. Bronner, S. Müller, R. Kiefer, R. Quay, M. Mikulla, O. Ambacher, A. Graff, F. Altmann, M. Simon, Mater. Sci. Forum 725, 79 (2012).

[6] R.J.T. Simms, F. Gao, Y. Pei, T. Palacios, U.K. Mishra, M. Kuball, Appl. Phys. Lett. 97, 033502 (2010).

[7] F. Gütle, V.M. Polyakov, M. Baeumler, F. Benkhelifa, S. Müller, M. Dammann, M. Cäsar, R. Quay, M. Mikulla, J. Wagner, O. Ambacher, Semicond. Sci. Technol. 27, 125003 (2012) and Ref. therein.

[8] V. Palankovski, S. Vitanov, R. Quay, in: IEEE Electron Devices Society: IEEE Compound Semiconductor Integrated Circuit Symp., CSICS 2006, CD-ROM: San Antonio (Texas, USA), IEEE, Piscataway, NJ 2006, p. 107.

[9] J. Joh, F. Gao, T. Palacios, J.A. del Alamo, Microelectron. Reliab. 50, 767 (2010).

[10] J. Wuerfl, E. Bahat-Treidel, F. Brunner, E. Cho, O. Hilt, P. Ivo, A. Knauer, P. Kurpas, R. Lossy, M. Schulz, S. Singwald, M. Weyers, R. Zhytnytska, Microelectron. Reliab. 51, 1710 (2011).

[11] S.Y. Park, C. Floresca, U. Chowdhury, J.L. Jimenez, C. Lee, E. Beam, b, P. Saunier, T. Balistreri, M.J. Kim, Microelectron. Reliab. 49, 478 (2009).

[12] M. Dammann, M. Baeumler, F. Gütle, M. Cäsar, H. Walcher, P. Waltereit, W. Bronner, S. Müller, R. Kiefer, R. Quay, M. Mikulla, O. Ambacher, A. Graff, F. Altmann, in: IEEE Int. Integrated Reliability Workshop, IRW 2011, Final report, S. Lake Tahoe, California USA, 2011, IEEE, Piscataway, NJ 2011, p. 42.

[13] W. Saito, in: Proc. IEEE International Reliability Physics Symposium (IRPS 2011), IEEE, Monterey 2011, p. 418 and references therein.

[14] ATLAS User's Manual 2005, Device Simulation Software, Silvaco International.

[15] M. Dammann, M. Baeumler, F. Gütle, M. Wespel, R. Reiner, V. Polyakov, P. Waltereit, R. Quay, M. Mikulla, O. Ambacher, Proc. 37th Workshop on Compound Semiconductor Devices and Integrated Circuits held in Europe (WOCSDICE 2013), Warnemünde, p. 190.

[16] D. Jin, J.A. del Alamo, in: Proc. 24th Int. Symp. on Power Semiconductor Devices and ICs (ISPSD), IEEE, Bruges 2012, p. 333.

[17] F. Benkhelifa, D. Krausse, S. Müller, R. Quay, M. Mikulla, O. Ambacher, in: Proc. 5th Space Agency-MOD (ESAMOD 2010), Round Table Workshop on GaN Component Technologies ESA, ESA Publications Division, Noordwijk 2010.

[18] K. Turvey, J. Allen, J. Phys. C 6, 2887 (1973) and references therein.

[19] S. Ganguly, J. Verma, G.W. Li, T. Zimmermann, H.L. Xing, D. Jena, Appl. Phys. Lett. 99, 193504 (2011).

[20] N. Onojima, M. Higashiwaki, J. Suda, T. Kimoto, T. Mimura, T. Matsui, J. Appl. Phys. 101, 043703 (2007). 